\title{
Volume Oscillations Delivered to a Lung Model Using 4 Different Bubble CPAP Systems
}

\author{
Jonathan A Poli, C Peter Richardson PhD, and Robert M DiBlasi RRT-NPS FAARC
}

\begin{abstract}
BACKGROUND: High-frequency pressure oscillations created by gas bubbling through an underwater seal during bubble CPAP may enhance ventilation and aid in lung recruitment in premature infants. We hypothesized that there are no differences in the magnitude of oscillations in lung volume $(\Delta V)$ in a preterm neonatal lung model when different bubble CPAP systems are used. METHODS: An anatomically realistic replica of an infant nasal airway model was attached to a Silastic test lung sealed within a calibrated plethysmograph. Nasal prongs were affixed to the simulated neonate and supported using bubble CPAP systems set at $6 \mathrm{~cm} \mathrm{H}_{2} \mathrm{O} . \Delta \mathrm{V}$ was calculated using pressure measurements obtained from the plethysmograph. RESULTS: The Fisher \& Paykel Healthcare bubble CPAP system provided greater $\Delta V$ than any of the other devices at all of the respective bias flows $(P<.05)$. The Fisher $\&$ Paykel Healthcare and Babi.Plus systems generally provided $\Delta \mathrm{V}$ at lower frequencies than the other bubble CPAP systems. The magnitude of $\Delta \mathrm{V}$ increased at bias flows of $>4 \mathrm{~L} / \mathrm{min}$ in the Fisher \& Paykel Healthcare, Airways Development, and homemade systems, but appeared to decrease as bias flow increased with the Babi.Plus system. CONCLUSIONS: The major finding of this study is that bubble CPAP can provide measureable ventilation effects in an infant lung model. We speculate that the differences noted in $\Delta \mathbf{V}$ between the different devices are a combination of the circuit/nasal prong configuration, bubbler configuration, and frequency of oscillations. Additional testing is needed in spontaneously breathing infants to determine whether a physiologic benefit exists when using the different bubble CPAP systems. Key words: bubble CPAP; respiratory distress syndrome; noninvasive ventilation; lung model; nasal prongs; flow dependence. [Respir Care 2015;60(3):371-381. (C) 2015 Daedalus Enterprises]
\end{abstract}

\section{Introduction}

A common treatment for spontaneously breathing infants with respiratory distress syndrome involves the application of bubble CPAP. ${ }^{1}$ Bubble CPAP is a simple and

\footnotetext{
Mr Poli, Dr Richardson, and Mr DiBlasi are affiliated with the Center for Developmental Therapeutics, Seattle Children's Hospital Research Institute, Seattle, Washington. Dr Richardson is also affiliated with the University of Washington School of Medicine, Seattle, Washington. Mr DiBlasi is also affiliated with the Respiratory Care Department, Children's Hospital and Regional Medical Center, Seattle, Washington.
}

Supplementary material related to this paper is available at http:// www.rcjournal.com.

The study was supported by the Respiratory Care Department and the Center for Developmental Therapeutics of Seattle Children's Hospital Research Institute. Mr Poli and Dr Richardson have disclosed no conflicts of interest. Mr DiBlasi has relationships with Dräger, Ikaria, and Vapotherm. inexpensive form of CPAP that has been used for nearly 4 decades to support infants with all forms of respiratory distress. ${ }^{1}$ Unlike CPAP provided by a mechanical ventilator, bubble CPAP transmits small-amplitude, highfrequency pressure oscillations around the mean airway pressure $\left(\overline{\mathrm{P}}_{\mathrm{aw}}\right) .^{2}$ These pressure oscillations are created by gases bubbling through the air-water interface of the submerged expiratory tube. Lee et $\mathrm{al}^{3}$ first observed the chest walls of infants supported by bubble CPAP oscillating at a frequency similar to high-frequency oscillatory ventila-

\footnotetext{
Mr Poli presented a version of this paper at the AARC Congress 2010, held December 6-8, 2010, in Las Vegas, Nevada.

Correspondence: Jonathan Poli, Center for Developmental Therapeutics, Seattle Children's Hospital Research Institute, 1900 Ninth Avenue, Seattle, WA 98101. E-mail: jpoli43@gmail.com.
}

DOI: $10.4187 /$ respcare. 03432 
tion. Furthermore, Pillow et $\mathrm{al}^{2}$ demonstrated that mechanical pressure oscillations created by bubble CPAP may be more beneficial than ventilator CPAP to aid in lung recruitment and to improve gas exchange in premature lambs, claiming that oscillations augment ventilation.

Due to its low cost and simplicity, institutions have used off-label bubble CPAP devices constructed from materials that are commonly found in the hospital setting. ${ }^{4}$ Recently, medical device manufacturers have received FDA clearances to market bubble CPAP systems designed to provide respiratory support for infants. Because fluid dynamics are sensitive to geometric and mechanical properties of the entire bubble CPAP system, the pressure waveforms are likely to be different; in other words, the $\overline{\mathrm{P}}_{\mathrm{aw}}$ and oscillatory effects may be different depending on the device, circuits, and patient interfaces used. According to previous studies in vitro, the $\overline{\mathrm{P}}_{\mathrm{aw}}$ and magnitude of airway pressure $\left(\mathrm{P}_{\mathrm{aw}}\right)$ oscillations will increase in size and frequency as bias flow is increased ${ }^{5}$; nevertheless, these parameters have not been rigorously measured in manufactured systems using a realistic infant airway model. We hypothesized that there are no differences in the magnitude of oscillations in lung volume $(\Delta \mathrm{V})$ and dominant frequencies of oscillations among 4 bubble CPAP systems.

\section{Methods}

\section{Bubble CPAP Systems}

We evaluated the 4 bubble CPAP systems shown in Figure 1: the homemade system (Fig. 1A) that has been described by several investigators ${ }^{4,6,7}$ and 3 FDA-cleared systems: bubble CPAP system (Fisher \& Paykel Healthcare, Auckland, New Zealand), Babi.Plus bubble positive airway pressure valve with an $n$ CPAP nasal kit (A Plus Medical, Carlsbad, California), and WaterPAP (Airways Development, Kenilworth, New Jersey) (Fig. 1B). The

\section{QUICK LOOK}

\section{Current knowledge}

Bubble CPAP is a common treatment for spontaneously breathing infants with respiratory distress syndrome. Bubble CPAP creates a mean airway pressure along with oscillatory effects that may be different depending on the device, circuit, and interface.

\section{What this paper contributes to our knowledge}

The pressure oscillations, volume oscillations, dominant frequency, and frequency range varied significantly between the 4 bubble CPAP systems tested. These variations are likely caused by a combination of the circuit/ nasal prong configuration, bubbler configuration, and frequency of oscillations. Clinical implications of these findings need to be elucidated.

homemade and Airways Development systems were equipped with Hudson RCI No. 2 prongs (catalog No. 1692, Teleflex Medical, Research Triangle Park, North Carolina), the Fisher \& Paykel Healthcare system was equipped with proprietary prongs and nasal interface (BC4030-10 and BC190-5, Fisher \& Paykel Healthcare), and the Babi.Plus system was equipped with No. 1 proprietary prongs (catalog No. 1010, A Plus Medical).

The homemade generator is a water-filled bottle with a corrugated tube submerged within it. The expiratory limb was stabilized in the water column using a $10-\mathrm{mL}$ syringe plunger; the CPAP level was determined by the length of the tube submerged in the water, indicated by tick marks at $1-\mathrm{cm}$ intervals written on the limb. ${ }^{7}$ The Airways Development generator is a similar design, but the plastic lid consists of a precut indent intended to hold the corrugated tubing in place. The Fisher \& Paykel Healthcare system
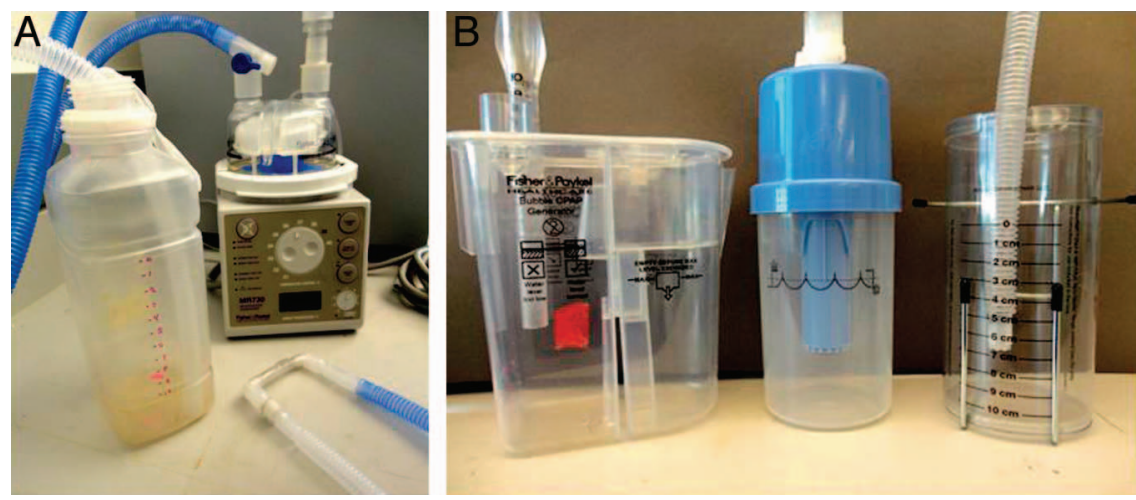

Fig. 1. Bubble CPAP systems used in the study. A: The homemade system equipped with the universal circuit (15-mm corrugated tubing and a Fisher \& Paykel Healthcare MR730 humidifier). B: The 3 FDA-cleared bubble CPAP systems from Fisher \& Paykel Healthcare (left), Babi.Plus (center), and Airways Development (right). 


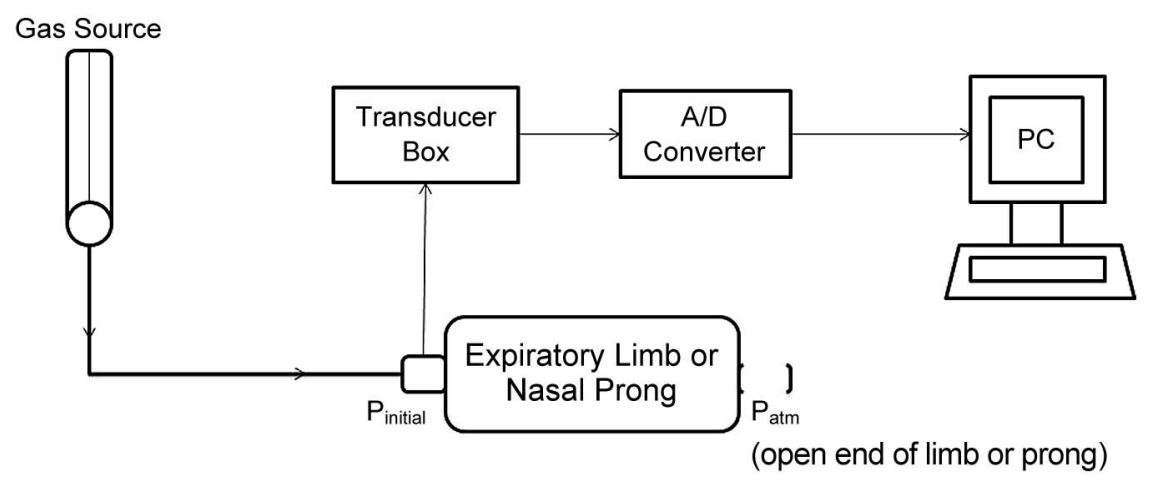

Fig. 2. Experimental apparatus for determining resistance of the expiratory limb and nasal prongs. $A / D=$ analog-to-digital; $P C=$ personal computer; $\mathrm{P}_{\text {initial }}=$ initial pressure; $\mathrm{P}_{\text {atm }}=$ atmospheric pressure.

has a rigid and ribbed polystyrene tube molded to fit inside the pressure generator. The Babi.Plus pressure generator expiratory limb possesses a rotation mechanism to change depth and is adjusted by turning a knob on the top of the lid. All of the systems were equipped with a standard 15-mm corrugated tubing circuit (BC-153, Fisher \& Paykel Healthcare) and the Fisher \& Paykel Healthcare MR730 humidifier shown in Figure 1A.

The water chamber cross-sectional area was determined at the surface and measured with digital calipers (catalog No. 500-197-30, Mitutoyo America, Aurora, Illinois). Similarly, the inside diameters of the expiratory limb and binasal prongs were determined using calipers. The resistance of the expiratory limb was determined by measuring pressure relative to atmosphere before expiratory limb and distal pressure (atmospheric pressure) at flows of 4, 6, 8, and $10 \mathrm{~L} / \mathrm{min}(n=3)$ (Fig. 2). Using the following equation of motion for a constant laminar flow system, pressure changes $(\Delta \mathrm{P})$ were plotted against bias flow, and the slope of the regression line was the determined as resistance:

$$
\Delta \mathrm{P}=\mathrm{P}_{\text {initial }}-\mathrm{P}_{\text {atm }}=\mathrm{P}_{\text {initial }}-0=\text { resistance } \times \text { flow }
$$

\section{Nasal Airway/Lung Model}

Nasal resistance in the newborn accounts for nearly half of the total airway resistance. ${ }^{8}$ As such, we designed a realistic replica of the nasal airway modeled from a computed tomography scan of an infant at 28 weeks of gestation. This model has been described in detail elsewhere. ${ }^{9}$

The lung model consisted of a Silastic infant test lung (compliance of $0.47 \mathrm{~mL} / \mathrm{cm} \mathrm{H}_{2} \mathrm{O}$ and resistance of $150 \mathrm{~cm} \mathrm{H}_{2} \mathrm{O} / \mathrm{L} / \mathrm{s}$; model 191, Maquet, Wayne, New Jersey) positioned inside a plethysmograph (14-cm diameter $\times 15$-cm height; IngMar Medical, Pittsburgh, Pennsylvania) that was surrounded by steel wool to maintain isothermal conditions. The plethysmograph was calibrated to enable conversion of pressure changes inside the plethysmograph to changes in volume of the Silastic test lung. Plethysmograph pressures were measured after adding known volumes of gas $(0,4,8,12$, and $16 \mathrm{~mL})$ to the test lung. A linear regression through the volume versus pressure data resulted in a calibration factor of $1.82 \mathrm{~mL} / \mathrm{cm} \mathrm{H}_{2} \mathrm{O}$ $\left(\mathrm{r}^{2}=0.10\right)$.

\section{Instrumentation}

Micromachined piezoresistive silicon pressure transducers (XRA515GN, Honeywell, Morristown, New Jersey) for measuring $\mathrm{P}_{\mathrm{aw}}$ and plethysmograph pressure were calibrated using 2-point calibrations with a precalibrated manometer (PM-23, Digitron, Devon, United Kingdom). Analog outputs from the $\mathrm{P}_{\mathrm{aw}}$ and plethysmograph pressure were sampled at $1,024 \mathrm{~Hz}$ using a 16-bit analog-to-digital converter (DT9804-EC-I-BNC, Data Translation, Marlborough, Massachusetts) and recorded on a personal computer. Pressure signals were recorded digitally, and calculations of $\overline{\mathrm{P}}_{\mathrm{aw}}$, amplitude of oscillations in $\mathrm{P}_{\mathrm{aw}}\left(\Delta \mathrm{P}_{\mathrm{aw}}\right)$ and plethysmograph pressure, and peak frequency of oscillations were made using custom software (Visual Basic, Microsoft, Redmond, Washington). For algorithms, please see the supplementary materials at http://www.rcjournal.com.

\section{Measuring Pressure Oscillations}

The nasal prongs were inserted into a Tygon tubing adapter that was affixed to the nasal airway model. The adapter formed a tight seal (no leak) between the nasal model and prongs. All bubble CPAP systems had the expiratory limb set to a depth of $6 \mathrm{~cm}$. Measurements of $\mathrm{P}_{\mathrm{aw}}$ were made downstream of the nasal airway model inside the prongs, and plethysmograph pressure was measured at the base of the plethysmograph. Using the homemade system with Hudson RCI prongs, bias flow was varied from 


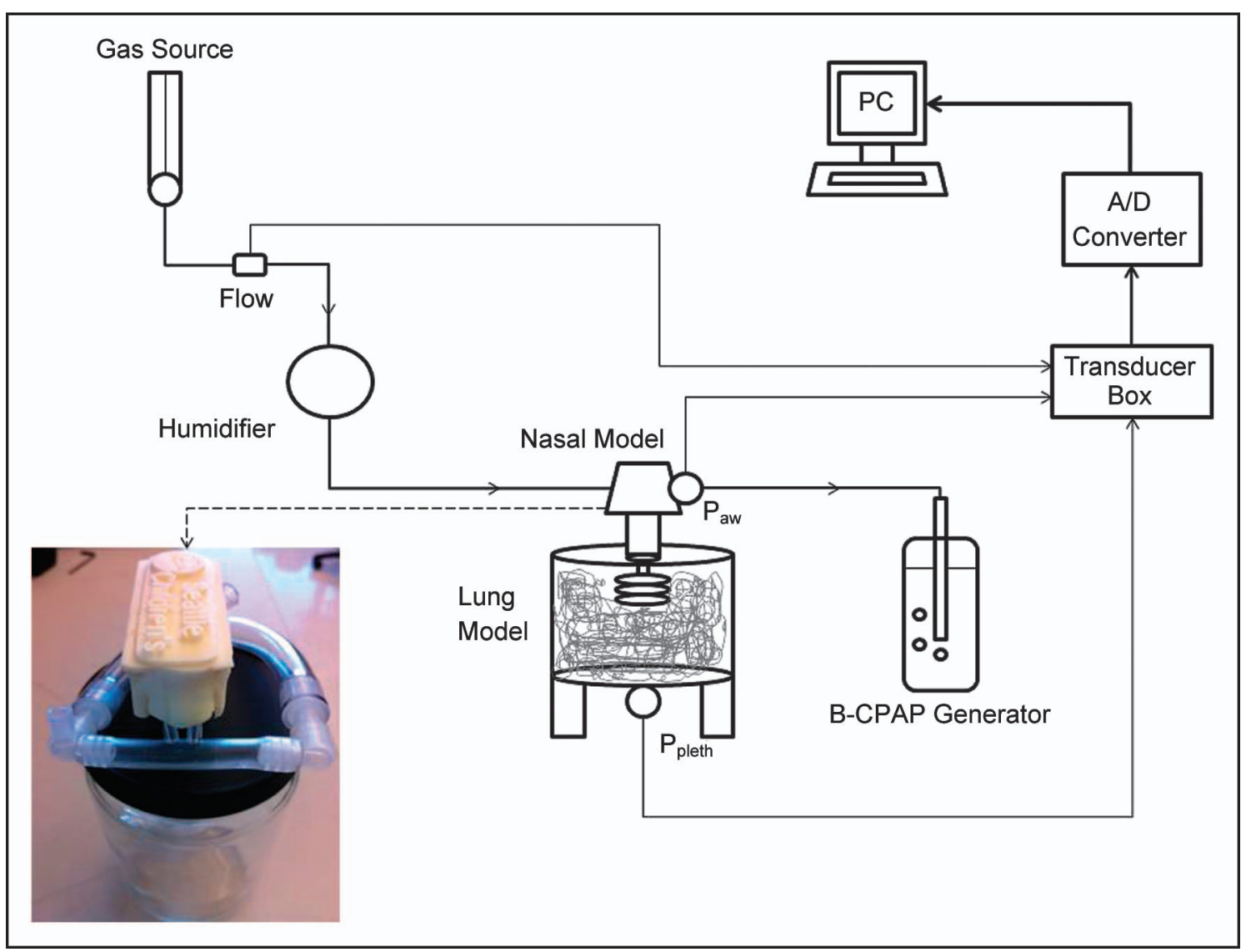

Fig. 3. Experimental apparatus for determining the magnitude of pressure oscillations. The nasal airway model is shown on the bottom left with Hudson $\mathrm{RCl}$ prongs. $\mathrm{PC}=$ personal computer; $\mathrm{A} / \mathrm{D}=$ analog-to-digital; $\mathrm{P}_{\mathrm{aw}}=$ airway pressure; $\mathrm{P}_{\text {pleth }}=$ plethysmograph pressure; $\mathrm{B}-\mathrm{CPAP}=$ bubble CPAP.

$2,4,6,8,10$, and $12 \mathrm{~L} / \mathrm{min}$, and $\mathrm{P}_{\mathrm{aw}}$ and plethysmograph pressure were recorded for $64 \mathrm{~s}$ at each flow; the bias flows were chosen to follow in the footsteps of previous studies. ${ }^{10,11}$ The Airways Development system was tested next, without changing the prongs. The same procedure was used for the Fisher \& Paykel Healthcare and Babi.Plus systems, interchanging the respective prongs per device. The experimental setup is shown in Figure 3.

\section{Data Analysis}

The sampled data were saved to a spreadsheet (Excel, Microsoft). The mean \pm SD of the airway oscillations $\left(\Delta \mathrm{P}_{\mathrm{aw}}\right)$ and volume oscillations $(\Delta \mathrm{V})$ were calculated using an algorithm (see the supplementary materials at http:// www.rcjournal.com) that determines an individual oscillation, the amplitude of the oscillation, and the mean of the oscillation amplitudes calculated throughout a recording session. $\overline{\mathrm{P}}_{\mathrm{aw}}$ was calculated by averaging the pressure from the 64-s run. Spectral power analysis was used to determine frequencies persisting in the system, and the dominant frequency was selected as the frequency with the maximum power observed. Due to the noisy nature of the bubble CPAP waveform, frequency range was recorded and determined by including all frequencies with a power of $\geq 100 \mathrm{~cm} \mathrm{H}_{2} \mathrm{O}^{2}$ in the range. Differences in $\mathrm{P}_{\mathrm{aw}}$ and $\Delta \mathrm{V}$ were first compared at the respective flows between devices and then within each device at the different flows using one-way analysis of variance for repeated measures with the Student-Newman-Keuls for post hoc analysis. Statistical significance was set a priori at $P<.05$.

\section{Results}

\section{Resistance of Expiratory Limb and Nasal Prong Interfaces}

Table 1 shows the respective mechanical properties of each component. The homemade device possessed the largest cross-sectional area within the group, whereas the Airways Development system possessed the smallest. The Babi.Plus expiratory limb had the largest inner diameter, whereas the Airways Development and Fisher \& Paykel Healthcare systems had the smallest. The Airways Devel- 
Table 1. Specifications and Mechanical Properties of Bubble CPAP Systems

\begin{tabular}{|c|c|c|c|c|c|}
\hline System & $\begin{array}{l}\text { Water Chamber } \\
\text { Cross-Sectional } \\
\text { Area at Surface } \\
\qquad\left(\mathrm{cm}^{2}\right)\end{array}$ & $\begin{array}{l}\text { Expiratory } \\
\text { Tube ID } \\
(\mathrm{mm})\end{array}$ & $\begin{array}{l}\text { Expiratory Tube } \\
\text { Resistance } \\
\left.\text { (cm } \mathrm{H}_{2} \mathrm{O} / \mathrm{L} / \mathrm{min}\right)\end{array}$ & Prong Type & $\begin{array}{l}\text { Prong Resistance } \\
\left.\text { ( } \mathrm{cm} \mathrm{H}_{2} \mathrm{O} / \mathrm{L} / \mathrm{min}\right)\end{array}$ \\
\hline Homemade & 64 & 12 & $0.032 \pm 0.003$ & $\begin{array}{l}\text { Hudson RCI infant nasal prongs } \\
\text { (No. 1, 2.8-mm ID) }\end{array}$ & $0.601 \pm 0.004$ \\
\hline Airways Development & 42 & 10 & $0.059 \pm 0.001$ & $\begin{array}{l}\text { Hudson RCI infant nasal prongs } \\
\text { (No. } 1,2.8 \text {-mm ID) }\end{array}$ & $0.601 \pm 0.004$ \\
\hline Fisher \& Paykel & 44 & 10 & $0.034 \pm 0.002$ & $\begin{array}{l}\text { Fisher \& Paykel FlexiTrunk } \\
\text { infant interface with bi-nasal } \\
\text { prongs (No. 4030, 3-mm ID) }\end{array}$ & $0.210 \pm 0.008$ \\
\hline Babi.Plus & 44 & 22 & $0.038 \pm 0.004$ & $\begin{array}{l}\text { Babi.Plus infant nasal prong } \\
\text { (No. 2, 3-mm ID) }\end{array}$ & $0.165 \pm 0.002$ \\
\hline diameter & & & & & \\
\hline
\end{tabular}

opment expiratory limb recorded the largest mean resistance, whereas the homemade expiratory limb recorded the lowest. Of the 3 nasal prong interfaces used, the Hudson RCI interface recorded the largest mean resistance, whereas the Babi.Plus interface recorded the lowest within the group.

\section{$\mathbf{P}_{\text {aw }}$ Oscillations}

Figure 4A shows pressure oscillations measured inside the nasal prongs as bias flow was increased from 2 to $12 \mathrm{~L} / \mathrm{min}$. When bias flow increased, the homemade, Fisher \& Paykel Healthcare, and Airways Development systems generated progressively larger $\Delta \mathrm{P}_{\mathrm{aw}}(P<.001)$. In contrast, the Babi.Plus system generated progressively smaller $\Delta \mathrm{P}_{\text {aw }}$ as flow increased from 2 to $8 \mathrm{~L} / \mathrm{min}(P=.043)$; at flows of $\geq 8 \mathrm{~L} / \mathrm{min}$, the magnitude of oscillations did not change $(P=.11)$. At all flows, the homemade and Fisher \& Paykel Healthcare devices generated larger $\Delta \mathrm{P}_{\mathrm{aw}}$ than the Airways Development and Babi.Plus systems $(P<.001)$. Between the homemade and Fisher \& Paykel Healthcare devices, at flows of $\leq 4 \mathrm{~L} / \mathrm{min}$, the Fisher \& Paykel Healthcare system generated greater $\Delta \mathrm{P}_{\mathrm{aw}}$ than the homemade system $(P=.02)$, but for flows of $>4 \mathrm{~L} / \mathrm{min}$, the homemade system generated greater $\Delta \mathrm{P}_{\text {aw }}(P<.001)$. The Airways Development system generated greater $\Delta \mathrm{P}_{\text {aw }}$ than the Babi.Plus system at all flows $(P<.001)$.

\section{Volume Oscillations}

Figure 4B shows volume oscillations as a function of bias flow. The homemade and Airways Development systems generated progressively larger $\Delta \mathrm{V}$ as flow increased $(P=.049)$. The magnitude of $\Delta \mathrm{V}$ increased with the Fisher $\&$ Paykel Healthcare system as the flow was increased from 2 to $4 \mathrm{~L} / \mathrm{min}(P<.001)$ but remained unchanged for flows of $>4 \mathrm{~L} / \mathrm{min}(P=.12)$. The Babi.Plus system exhibited a similar trend to the airway oscillations; the mag- nitude of $\Delta \mathrm{V}$ decreased as flow was adjusted from 2 to $8 \mathrm{~L} / \mathrm{min}(P=.044)$ but remained unchanged for flows of $>8 \mathrm{~L} / \mathrm{min}(P=.20)$. The Fisher \& Paykel Healthcare system generated greater $\Delta \mathrm{V}$ than all of the other systems at all flows $(P<.001)$. The homemade system generated the next greatest $\Delta \mathrm{V}$, which was greater than that generated by the Airways Development and Babi.Plus systems at all flows $(P<.001)$. There were no differences in $\Delta \mathrm{V}$ between the Airways Development and Babi.Plus systems at flows of $\leq 4 \mathrm{~L} / \mathrm{min}(P=.19)$, but for flows of $>4$ $\mathrm{L} / \mathrm{min}$, the Airways Development system generated larger $\Delta \mathrm{V}(P<.001)$

\section{Mean CPAP, Dominant Frequency, Frequency Range, and Waveform Attenuation}

Table 2 provides the mean CPAP level at each bias flow for each device. All systems increased mean CPAP with increasing flow. The largest change in the CPAP level was displayed by the Babi.Plus system, increasing by a total of $1.2 \mathrm{~cm} \mathrm{H}_{2} \mathrm{O}$ from 5.7 to $6.9 \mathrm{~cm} \mathrm{H}_{2} \mathrm{O}$ throughout the range of flows. The Airways Development system followed, increasing by a total of $0.9 \mathrm{~cm} \mathrm{H}_{2} \mathrm{O}$ from 6.3 to $7.2 \mathrm{~cm} \mathrm{H}_{2} \mathrm{O}$. The third largest change in the CPAP level was obtained with the Fisher \& Paykel Healthcare system, increasing by $0.8 \mathrm{~cm} \mathrm{H}_{2} \mathrm{O}$ from 5.9 to $6.7 \mathrm{~cm} \mathrm{H}_{2} \mathrm{O}$. The smallest increase in CPAP pressure was exhibited by the homemade system, increasing by $0.6 \mathrm{~cm} \mathrm{H}_{2} \mathrm{O}$ from 5.8 to $6.4 \mathrm{~cm} \mathrm{H}_{2} \mathrm{O}$.

The dominant frequencies and frequency ranges generated by each device at each flow are given in Table 2 as well. With the homemade system, the dominant frequency increased from $7 \mathrm{~Hz}$ at $2 \mathrm{~L} / \mathrm{min}$ flow to a dominant frequency maximum of $20 \mathrm{~Hz}$ at $10 \mathrm{~L} / \mathrm{min}$ and then fell to $15 \mathrm{~Hz}$ at $12 \mathrm{~L} / \mathrm{min}$. The Airways Development system maintained a dominant frequency of $6-7 \mathrm{~Hz}$ at $2-8 \mathrm{~L} / \mathrm{min}$, but at $10 \mathrm{~L} / \mathrm{min}$, the system increased to $14 \mathrm{~Hz}$ and fell to $11 \mathrm{~Hz}$ at $12 \mathrm{~L} / \mathrm{min}$. The Babi.Plus and Fisher \& Paykel 


\section{Volume Oscillations in 4 Different Bubble CPAP Systems}
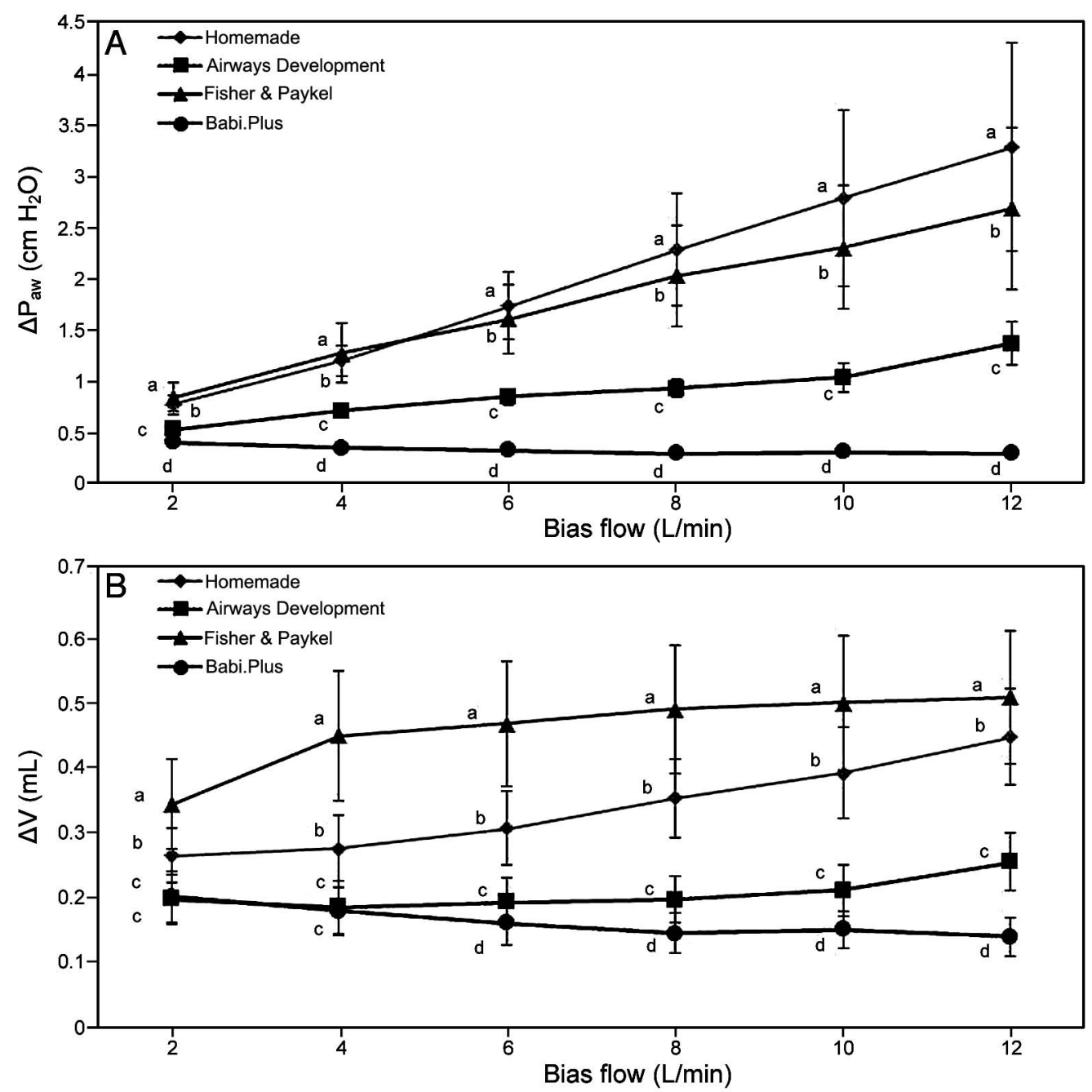

Fig. 4. A: Average size of airway oscillations $\left(\Delta P_{a w}\right)$. B: lung volume oscillations $(\Delta V)$ as a function of bias flow. Measurements were taken at a set depth level of $6 \mathrm{~cm}$. Data are shown as mean \pm SD. Letters above the data points denote a statistical order of the largest mean (a) to the smallest mean (d) at that particular bias flow. In the 2 cases in which $\Delta \mathrm{V}$ has the same letter (c), the means are statistically not different $(P>.05)$ as a result of the Student-Newman-Keuls analysis; otherwise, the means are statistically different $(P<.05)$.

Table 2. Mean CPAP, Dominant Frequency, and Frequency Range for Each Bubble CPAP System at Each Flow

Bias Flow (L/min)

$\begin{array}{llllll}2 & 4 & 6 & 10 & 12\end{array}$

$\mathrm{CPAP}$, mean $\pm \mathrm{SD}$ $\left(\mathrm{cm} \mathrm{H}_{2} \mathrm{O}\right)$

Homemade Fisher \& Paykel Airways Development

$5.76 \pm 0.08$

$5.90 \pm 0.06$

$5.84 \pm 0.16$

$6.31 \pm 0.05$

$6.01 \pm 0.11$

$5.72 \pm 0.01$

$6.48 \pm 0.07$

$5.91 \pm 0.01$

Babi.Plus

Dominant frequency

(frequency range, $\mathrm{Hz}$ )

Homemade

Fisher \& Paykel

Airways Development

Babi.Plus

$\begin{array}{ccc}7.25(3.5-9.5) & 9.50(3.5-35) & 14.78(3-35) \\ 5.60(4-7) & 6.20(3-9,22-34) & 7.00(2-38) \\ 6.16(4.5-7.5) & 6.47(5-9) & 6.86(4-15.5) \\ 6.86(4.5-8) & 5.78(4.5-8.5) & 5.45(4.5-7.5)\end{array}$

$7.25(3.5-9.5)$

$6.86(4.5-8)$

$\begin{array}{ll}5.95 \pm 0.21 & 6.08 \pm 0.26 \\ 6.14 \pm 0.15 & 6.29 \pm 0.17 \\ 6.64 \pm 0.09 & 6.81 \pm 0.09 \\ 6.17 \pm 0.01 & 6.46 \pm 0.01\end{array}$

$16.06(3-35,55-72)$

$6.53(2-38,62-66.5)$

$6.67(6-13)$

$5.65(4.5-8.5)$

$$
\begin{aligned}
& 6.20 \pm 0.29 \\
& 6.44 \pm 0.19 \\
& 6.99 \pm 0.11 \\
& 6.62 \pm 0.01
\end{aligned}
$$

$6.36 \pm 0.32$

$6.67 \pm 0.22$

$7.21 \pm 0.12$

$6.93 \pm 0.01$ 

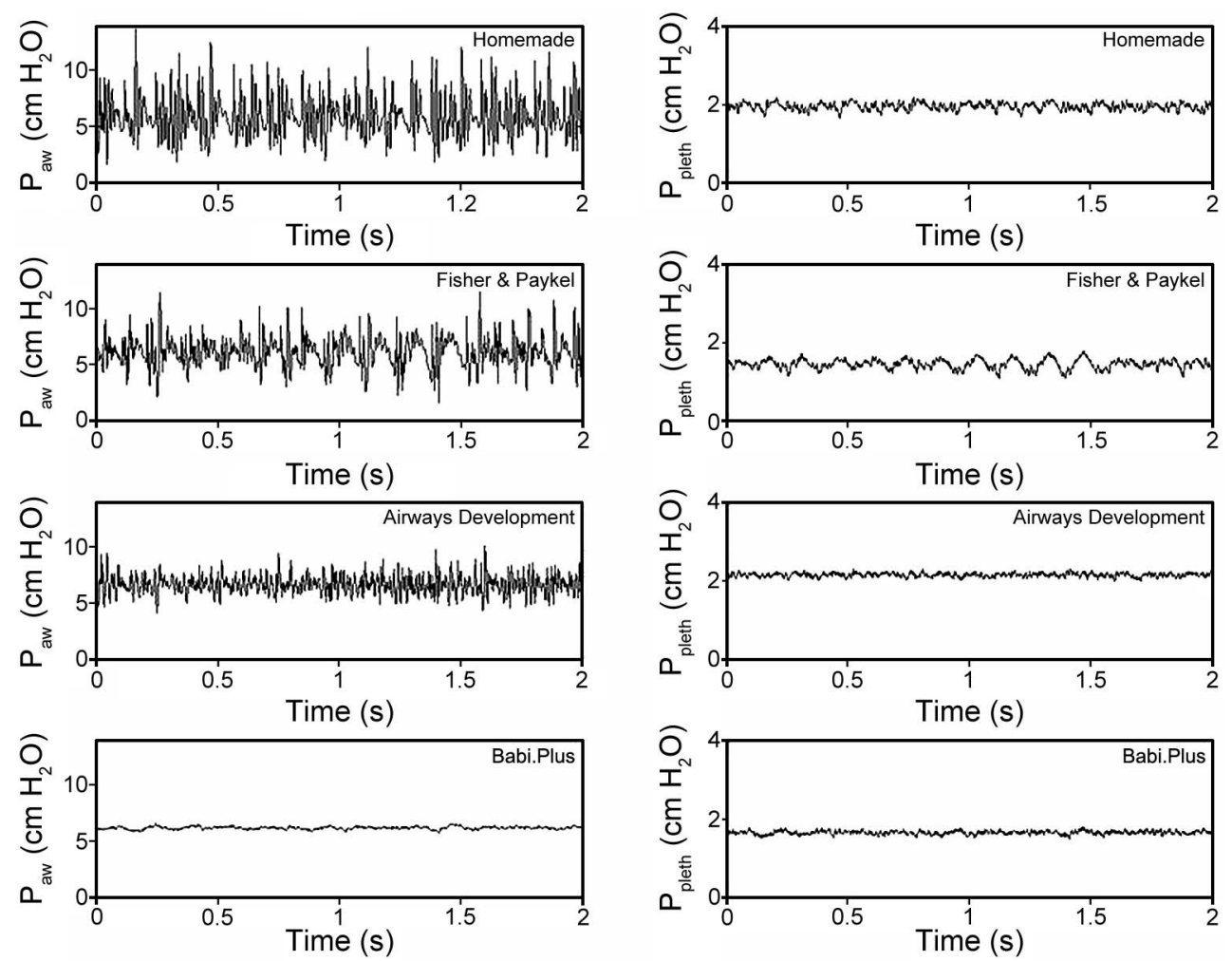

Fig. 5. The pressure waveform recorded at the nasal prongs (left) and plethysmograph (right) for each respective device. The depth was set at $6 \mathrm{~cm}$, and the bias flow was set at $6 \mathrm{~L} / \mathrm{min}$. These graphs show a 2-s snippet of the $64-\mathrm{s}$ recording time to see more detail. $\bar{P}_{\text {aw }}=$ airway pressure; $\mathrm{P}_{\text {pleth }}=$ plethysmograph pressure.

Healthcare bubble CPAP systems maintained the dominant frequencies at $5-8 \mathrm{~Hz}$ throughout the flow range.

Table 2 provides the range of delivered frequencies beneath the dominant frequency values. The Fisher \& Paykel Healthcare and homemade systems delivered a small range of frequencies (4-7 and 3.5-9.5 Hz, respectively) at 2 $\mathrm{L} / \mathrm{min}$. The range became larger with increasing bias flow, producing a bandwidth extending from 4 to $38 \mathrm{~Hz}$ for the Fisher \& Paykel Healthcare system and from 1.5 to $43 \mathrm{~Hz}$ for the homemade system. These systems saw an emergence of high frequencies at higher flows; the homemade and Fisher Paykel systems generated second bandwidths of 54-76 and $61-71 \mathrm{~Hz}$, respectively, accompanying the lower frequency bandwidth at flows of $\geq 8 \mathrm{~L} / \mathrm{min}$. The Airways Development system exhibited a similar increase in range of low frequencies, but not at as wide a range as the homemade or Fisher \& Paykel Healthcare system. At $2 \mathrm{~L} / \mathrm{min}$, the range was $4.5-7.5 \mathrm{~Hz}$ and maximized at $6-20.5 \mathrm{~Hz}$ at $12 \mathrm{~L} / \mathrm{min}$. No high-frequency bandwidth appeared for the Airways Development system. The Babi. Plus system did not increase the range of frequencies with increasing flow $(\sim 4-8 \mathrm{~Hz}$ at all flows) and did not produce a high-frequency bandwidth.
Figure 5 shows side by side the pressure waveforms measured in the airway (left) and plethysmograph (right). The homemade system generated the greatest variability (noise) in the $\mathrm{P}_{\mathrm{aw}}$ waveform, whereas the Babi.Plus system generated the least. The Fisher \& Paykel Healthcare system pressure waveform exhibited lower frequency pressure waves compared with the other systems. The Airways Development system appears as a flat CPAP line similar to the Babi.Plus system but with slightly more noise. Also, there was substantial attenuation in the magnitude of $\mathrm{P}_{\mathrm{aw}}$ and a reduction in variability between the $\mathrm{P}_{\mathrm{aw}}$ and plethysmograph pressure. The largest disparity in airway and plethysmograph pressure was observed with the homemade system. The Fisher \& Paykel Healthcare system lost the higher frequencies transitioning from the airway to the lung model, but the lower frequencies can be seen in the plethysmograph pressure waveform. The Airways Development system lost most of the noise variability and intensity from the airway to the lung. The Babi.Plus system exhibited the least change from the airway to the lung model; the plethysmograph pressure resembled a waveform similar to that obtained with the Airways Development system. 


\section{Discussion}

The major finding of this study is that the bubble CPAP systems provided quantifiable oscillations in $\Delta \mathrm{V}$ that may prove to be clinically meaningful and that the different bubble CPAP systems studied exhibited differences in the magnitude and frequency of volume oscillations delivered to a preterm infant lung model.

Bubble CPAP has been shown to increase oxygenation ${ }^{12}$ and rate of successful extubation ${ }^{13}$ while reducing apnea, ${ }^{6}$ hospital stay, ${ }^{14}$ and duration of mechanical ventilation ${ }^{15}$ in preterm infants. Because bubble CPAP is compared with conventional ventilator CPAP, ${ }^{12-15}$ the therapy's merits have been attributed to the oscillatory component and its ability to exploit nonlinear properties of the lung, inducing phenomena such as stochastic resonance that requires a nonlinear dynamic system (the lung), weak biological signal (CPAP), and superimposed noise (pressure oscillations). ${ }^{5}$ The connection was made by Suki and colleagues ${ }^{16-18}$ while studying variable ventilation in computer simulations and rodent models of acute lung injury. The noise in the pressure signal produced by the bubbling takes advantage of the nonlinearity of atelectatic regions of the lung by exploring higher volume values while mean pressure remains the same; these increases in volume are indicative of additional recruitment. ${ }^{19}$ All the devices in this study produced a noisy pressure signal. These waveforms may have mechanical effects, the observed motion in the lung caused by the Fisher \& Paykel Healthcare and homemade systems in particular, that could be clinically meaningful by acting as pneumatic pistons that can drive the lungs open, recruit airways, and move gas, or more subtly, the waveforms generated by the devices may be inducing stochastic resonance. Although these systems are all considered bubble CPAP systems, we have shown that there are distinct differences in the waveforms produced by these devices, and consequently, these devices could deliver different levels of respiratory support to patients. Understanding how particular design features influence the pressure waveform will distinguish these devices from one another with regard to their clinical impact and may guide future bubble CPAP designs depending on whether these features possess clinical merit.

A particular design feature that influences the pressure waveform present on two of the bubble CPAP systems is a diffuser component at the opening of the expiratory tube of the Babi.Plus and Airways Development systems. The diffuser component is a plastic barrier with small openings surrounding the edges. As air passes through the diffuser, the air-water seal is broken down into smaller sizes, creating smaller bubbles. The motivation for its incorporation is to reduce the oscillation magnitude to adhere to FDA regulations regarding a predicate positive airway pressure valve device (http://www.accessdata.fda.gov/cdrh_docs/ pdf9/k090317.pdf) Accessed November 12, 2014; the Babi.Plus and Airways Development systems fall under this distinction. Consequently, the oscillations generated by the Airways Development and Babi.Plus systems are lesser in magnitude compared with the Fisher \& Paykel Healthcare and homemade systems. Kahn et $\mathrm{al}^{10}$ and $\mathrm{Wu}$ et al ${ }^{11}$ performed in vitro studies testing the effects of changing the diameter of the expiratory limb on mean pressure, pressure range, and frequency and observed that although frequency and mean pressure decreased with increasing expiratory limb diameter, pressure range increased. Increasing limb diameter increases the area of the air-water seal, creating larger bubbles and larger swings in pressure. The diffuser decreases the water seal area by forcing the air column to partition while moving through it, muffling the pressure oscillations.

However, although both the Airways Development and Babi.Plus systems possess a diffuser, there is a difference in oscillation size between the systems. One distinction between the 2 generators is the size of the inside diameter of the expiratory limb and thus the attached diffuser; the diffuser on the Babi.Plus system is twice the size of that on the Airways Development system: 16 and 8 openings, respectively. The Babi.Plus diffuser breaks the air column into smaller bubbles, attenuating the oscillations more so than the Airways Development diffuser. Nevertheless, the behavior of the Babi.Plus system contradicts other studies $^{9,10,20}$ on bubble CPAP systems because it does not increase in oscillation size with increasing flow. However, a closer look shows that the Airways Development system initially decreases its volume oscillation size from 2 to $4 \mathrm{~L} / \mathrm{min}$. Because resistance is inversely related to the fourth power of radius, the back pressure created by the Airways Development expiratory limb may force the air through the diffuser at a rate that overpowers the diffuser's ability to break apart the air column at higher flows. In contrast, the Babi.Plus expiratory limb has a large enough radius to reduce oscillation size at higher flows, whereas the Airways Development generator can reduce oscillation size only at flows of $<6 \mathrm{~L} / \mathrm{min}$.

Another unanticipated finding was the exchange between the Fisher \& Paykel Healthcare and homemade systems with regard to airway and volume oscillations. The homemade system generated greater airway oscillations yet lesser lung oscillations than the Fisher \& Paykel Healthcare system. An uncommon denominator between the systems was the prongs used. Pillow et $\mathrm{al}^{5}$ documented a substantial attenuation of oscillation size transitioning from the airway to the model lung, suggesting that the resistance of the airway plays a role in reducing oscillation size. Therefore, because the nasal prongs are connected in series, the prong resistance must also play a role in reducing oscillation size. The Fisher \& Paykel Healthcare proprietary prongs 
have a smaller resistance than the Hudson RCI prongs. Thus, the higher resistance of the Hudson RCI prongs assists in attenuating the pressure oscillations on their way to the lung. The Babi.Plus proprietary prongs also have less resistance than the Hudson RCI prongs. As a result, the Airways Development system decreases oscillation magnitude more so than the less resistive Babi.Plus system, which is reflected in the similar volume oscillation size of the 2 devices at low flows $(<6 \mathrm{~L} / \mathrm{min})$.

The mean CPAP levels of the 3 systems did not differ substantially with increases in flow and were mainly linearly dependent. The homemade system exhibited the least increase in mean CPAP because it possessed the least resistive expiratory tube. The Fisher \& Paykel Healthcare system showed the second least increase in mean CPAP due to having the second least resistive expiratory tube. The largest swing in pressure was demonstrated by the Babi.Plus system, although its resistance was lower compared with the Airways Development expiratory tube. The larger diffuser may add a unique element to the CPAP pressure; the Babi.Plus system increased nonlinearly with regard to mean CPAP. Nevertheless, these results are not consistent with the results of Kahn et al, ${ }^{10}$ who, within a flow range of 4-12 L/min in a no-leak system, documented a change in mean CPAP of 3-4 $\mathrm{cm} \mathrm{H}_{2} \mathrm{O}$. Pillow et al documented a change of $3 \mathrm{~cm} \mathrm{H}_{2} \mathrm{O}$ at a flow range of $2-10 \mathrm{~L} / \mathrm{min}$ in a similar system. Our study documented, at most, a change of $1.2 \mathrm{~cm} \mathrm{H}_{2} \mathrm{O}$ delivered within a flow range of $2-12 \mathrm{~L} / \mathrm{min}$. The sophistication of the designs may account for the small increase in pressure, but in our study, the homemade system exhibited the least change in mean CPAP level. Those numbers are comparable to the data presented by Ho et al, ${ }^{21}$ who showed a change of $1.82 \mathrm{~cm} \mathrm{H}_{2} \mathrm{O}$ within the tubing system at the same flow range. Although there is still a need to monitor the CPAP level, the increase in pressure does not appear to be as alarming as previous studies warned. As one of the major arguments against implementation of bubble CPAP in the clinical setting, we think that more studies measuring the rise in mean CPAP with increases in flow need to be performed to establish clinical importance.

Dominant frequency output varied greatly for the homemade system, somewhat for the Airways Development system, and very little for the Babi.Plus and Fisher \& Paykel Healthcare systems. It appears that sophistication in engineering plays a role in the dominant frequency delivered to the patient. The Babi.Plus and Fisher \& Paykel Healthcare systems have more elaborate designs and therefore can deliver frequencies within a small range. Our results are comparable to previous studies using the Babi. Plus and Fisher \& Paykel Healthcare systems that measured values in dominant frequencies produced by these devices. ${ }^{5,20}$ One explanation is that the more sophisticated designs keep the expiratory tube in position with increas- ing flow. The distal end of the expiratory tube in the homemade system would sway horizontally with increasing flow; the extra momentum of the tube may contribute to the variation in frequencies. The Airways Development expiratory tube also possesses the same motion at high flows and thus its increase in dominant frequencies. An interesting implication of the Fisher \& Paykel Healthcare and Babi.Plus bubble CPAP generators is that there may be a way to design a bubble CPAP system to deliver predictable frequencies; the ability to deliver a noisy signal with a dominant frequency may facilitate a system to stochastic resonance. ${ }^{19}$ Our measurement system showed a profound transformation in the waveform as it moved through the airway, but the longer waves appear to have the most success passing through the airway model, and in particular, the Fisher \& Paykel Healthcare system maintained most of its longer waves though the transmission. Furthermore, increasing the flow for the homemade and Airways Development devices could decrease their oscillatory function because their dominant frequencies increase, thereby losing them to the mechanical filtering seen in other lung models. ${ }^{5}$

The composition of the pressure signal varies greatly between the devices; in particular, the homemade and Fisher \& Paykel Healthcare systems generate a more diverse signal compared with the Airways Development and Babi. Plus signals. Because of filtering, the pressure waveform changes drastically from the airway to the lung. If a pressure wave does not have enough energy, most likely it dissipates before reaching the lower airways and alveoli. Pressure wave energy comes from the interaction between the water and air; mean CPAP is a result of the hydrostatic depth, and the oscillations transmitted through the airway are a result of the bubbling. The diffuser present on the Babi.Plus and Airways Development system decreases interaction between the air and water. In contrast, the homemade and Fisher \& Paykel Healthcare systems have more interaction between the water and air with no diffuser. With more area between the air-water seal (the size of the bubble breaking off the tube), a larger amount of energy is transmitted to the pressure waves traveling back through the system into the patient. This phenomenon was discussed by DiBlasi et $\mathrm{al}^{22}$ in a study investigating highamplitude bubble CPAP in an attempt to explain the large $\Delta \mathrm{P}_{\text {aw }}$ observed in a $135^{\circ}$ expiratory limb configuration. As a result, by allowing more energy to transmit to the air column, there is a greater chance that more frequencies will receive sufficient energy to travel through the lung. The larger frequency ranges produced by the homemade and Fisher \& Paykel Healthcare systems attest to the distribution of energy. In contrast, the Babi.Plus and Airways Development systems produced lower ranges because the energy distributed to other frequencies was insufficient to pass through the airway. As a result, the role of the oscil- 


\section{Volume Oscillations in 4 Different Bubble CPAP Systems}

latory component is not as prevalent or noisy for the bubble CPAP devices with the diffuser compared with the ones without it.

\section{Limitations}

Although in vitro models have been shown to be effective surrogates for analyzing high-frequency oscillatory ventilation systems, ${ }^{23}$ the present model possesses similar limitations as discussed in previously published bench studies. $5,10,18,20$ A major limitation is that an in vitro model does not allow reliable correlations to patients due to its simplicity. Furthermore, our study did not have a leak in the system, so the oscillations will attenuate more than anticipated from the results in a realistic setting. The performance of these devices was not evaluated on each prong interface; therefore, it may be unclear whether some of the disparities are a result of the bubble CPAP generator or different resistive prongs. Finally, although anatomically similar, our airway model does not fully mimic the upper airway of a preemie. In particular, the material used for our model is not the same as the tissue that makes up the upper airway, and it lacks mucosa and other particulates that are present, thereby having a different effect on oscillation transmission. The limitations, however, illuminate several steps that must be taken to improve bubble CPAP experimentation. Studies performed with more realistic resistance models or in vivo models will provide more accurate estimates of oscillation magnitude. Our study has attempted to develop a more realistic nasal airway model by replicating the structure of the nasal passage and pharynx. Combined with a lung model that possesses a realistic compliance and obeys power law behavior, the mechanical filtering effect can be studied in detail, exposing the behavior of the pressure wave moving through the respiratory system.

\section{Clinical Implications}

Statistical differences between the devices can be attributed to the large number of observations made at each flow. However, although statistical differences exist, based on the limitations mentioned, it is impossible to extrapolate from these findings and expect a specific physiologic outcome. Clinicians monitoring $\mathrm{P}_{\mathrm{aw}}$ should approach these measurements with some trepidation because $\mathrm{P}_{\mathrm{aw}}$ is a poor surrogate for lung pressure during bubble CPAP.

Although most studies focus on premature infants, a randomized controlled trial studied a cohort of subjects at $\sim 36$ weeks of gestation placed on bubble CPAP or variable-flow CPAP. ${ }^{24}$ No differences were reported between the therapies for outcome measures of percentage CPAP failure, percentage barotrauma, duration of CPAP use, duration of oxygen use, ICU stay, and hospital stay. The findings suggest that the physiologic effect of the pressure oscillations from bubble CPAP is less prevalent as the lungs mature. In other words, as the infant reaches term age, the more compliant lung absorbs the oscillations as they propagate through the airways. This is consistent with data from in vitro models that measured effects of compliance change on oscillation magnitudes and showed marked decreases in size of pressure oscillations as well as frequencies. ${ }^{5,10} \mathrm{~A}$ broader review of the merit of bubble CPAP for infants at $>36$ weeks is needed to determine whether it should be used for a more mature patient population.

Although the homemade device is the most cost-effective form of bubble CPAP, it possesses more variability in its frequency output than the other devices. If employed in the clinical setting, patients on the homemade device will experience a different waveform at different flows. The variability may be attributed to the tube submerged inside the homemade system and the increase in swaying motion observed as flow increases. The Airways Development system has the same issue, but it has less variability compared with the homemade device due to its CPAP level adjustment mechanism. Based on cost, the more expensive devices, such as the Fisher \& Paykel Healthcare and Babi. Plus devices, have less variability than their cheaper counterparts. Knowing the exact pressure waveform being delivered to a patient is useful for a hospital standardizing care.

Furthermore, with regard to ergonomics and safety, the FDA-approved systems have the most reliable designs. The Babi.Plus bubble CPAP generator possesses the most elaborate engineering mechanism for CPAP level adjustments. Although ergonomically efficient, this also prevents drastic shifts in the CPAP level if mishandled. The Fisher \& Paykel Healthcare system utilizes indents on the expiratory limb to secure the CPAP level and possesses an intricate mechanism for maintaining consistent water level not found on the Babi.Plus system, but there is a risk of CPAP level change if mishandled and the expiratory limb changes depth suddenly. The Airways Development system utilizes a similar mechanism as the Fisher \& Paykel Healthcare system to adjust and secure the CPAP level, but it does not possess a mechanism for maintaining water level. Nevertheless, all of these devices come with a pressure relief component to prevent barotrauma from drastic increases in pressure. The homemade system secures its depth with a syringe plunger, which may not be the most reliable method of maintaining CPAP. Furthermore, the homemade system does not possess a pressure relief mechanism. As price increases, performance consistency, fullproofing the CPAP level, and safety against drastic pressure increases are included. Although the magnitude of oscillations may not be reflected in the cost of the systems or the hierarchy of designs, the clinical application of the 


\section{Volume Oscillations in 4 Different Bubble CPAP Systems}

systems benefits from the refined ergonomic utility with regard to performance and safety.

With regard to clinical practice, the best device would depend on the type of therapy a clinician wants to employ. If ventilator-like CPAP is best for the patient, then the Babi.Plus system would be the closest option, but if oscillatory therapy is desired for infants with low lung compliance, then the Fisher \& Paykel Healthcare system would result in the largest fluctuations in $\Delta \mathrm{V}$. Although each system uses a similar means to deliver bubble CPAP, the resulting therapy delivered to the patient may be different depending on which system is used.

\section{Conclusions}

Our data indicate that pressure oscillations, volume oscillations, dominant frequency, and frequency range differ among the devices, leading us to reject our original hypothesis. Although there have been studies in vitro and in vivo using the homemade, ${ }^{4,6}$ Babi.Plus, ${ }^{20}$ and Fisher \& Paykel Healthcare ${ }^{2,5}$ systems, this is the first study comparing systems with one another. Furthermore, this is the first study investigating the Airways Development system.

\section{ACKNOWLEDGMENTS}

We thank Christopher Howard MBA for collaboration during the protocol and Thomas Hansen MD and Charles Smith PhD for feedback and support.

\section{REFERENCES}

1. DiBlasi RM. Nasal continuous positive airway pressure (CPAP) for the respiratory care of the newborn infant. Respir Care 2009;54(9): 1209-1235.

2. Pillow JJ, Hillman N, Moss TJ, Polglase G, Bold G, Beaumont C, et al. Bubble continuous positive airway pressure enhances lung volume and gas exchange in preterm lambs. Am J Respir Crit Care Med 2007; 176(1):63-69.

3. Lee KS, Dunn MS, Fenwick M, Shennan AT. A comparison of underwater bubble continuous positive airway pressure with ventilator-derived continuous positive airway pressure in premature neonates ready for extubation. Biol Neonate 1998;73(2):69-75.

4. Aly H, Milner JD, Patel K, El-Mohandes AA. Does the experience with the use of nasal continuous positive airway pressure improve over time in extremely low birth weight infants? Pediatrics 2004; 114(3):697-702

5. Pillow JJ, Travadi JN. Bubble CPAP: is the noise important? An in vitro study. Pediatr Res 2005;57(6):826-830.

6. Chan KM, Chan HB. The use of bubble CPAP in preterm infants: local experience. HK J Pediatr 2007;12:86-92.

7. Bonner KM, Mainous RO. The nursing care of the infant receiving bubble cpap therapy. Adv Neonatal Care 2008;8(2):78-95.

8. Polgar G, Kong GP. The nasal resistance of newborn infants. J Pediatr 1965;67(4):557-567.
9. Youngquist TM, Richardson CP, DiBlasi RM. Effects of condensate in the exhalation limb of neonatal circuits on airway pressure during bubble CPAP. Respir Care 2013;58(11):1840-1846.

10. Kahn DJ, Courtney SE, Steele AM, Habib RH. Unpredictability of delivered bubble nasal continuous positive airway pressure: role of bias flow magnitude and nares-prong air leaks. Pediatr Res 2007; 62(3):343-347

11. Wu CS, Lee CM, Yuh YS, Hua YM. Influence of changing the diameter of the bubble generator bottle and expiratory limb on bubble CPAP: and in vitro study. Pediatr Neonatol 2012;53(6):359-365.

12. Courtney SE, Kahn DJ, Singh R, Habib RH. Bubble and ventilatorderived nasal continuous positive airway pressure in premature infants: work of breathing and gas exchange. J Perinatol 2011;31(1): 44-50.

13. Gupta S, Sinha SK, Tin W, Donn SM. A randomized controlled trial of post-extubation bubble continuous positive airway pressure versus infant flow driver continuous positive airway pressure in preterm infants with respiratory distress syndrome. J Pediatr 2009;154(5): 645-650.

14. Bahman-Bijari B, Malekiyan A, Niknafs P, Baneshi MR. BubbleCPAP vs. ventilatory-CPAP in preterm infants with respiratory distress. Iran J Pediatr 2011;21(2):151-158.

15. Tapia JL, Urzua S, Bancalari A, Meritano J, Torres G, Fabres J, et al. Randomized trial of early bubble continuous positive airway pressure for very low birth weight infants. J Pediatr 2012;161(1):75.e180.e1.

16. Suki B, Alencar AM, Sujeer MK, Lutchen KR, Collins JJ, Andrade JS, et al. Life-support system benefits from noise. Nature 1998; 393(6681):127-128.

17. Arold SP, Mora R, Lutchen KR, Ingenito EP, Suki B. Variable tidal volume ventilation improves lung mechanics and gas exchange in a rodent model of acute lung injury. Am J Respir Crit Care Med 2002;165(3):366-371.

18. Thammanomai A, Hueser LE, Majumdar A, Bartolák-Suki E, Suki B. Design of a new variable-ventilation method optimized for lung recruitment in mice. J Appl Phyisol 2008;104(5):1329-1340.

19. Suki B. Fluctuations and power laws in pulmonary physiology. Am J Respir Crit Care Med 2002;166(2):133-137.

20. Goldstein MR, Tong C, Merritt TA, Terry M, Tiras M, Fayard E, Peverini R. Can the frequency of the vibratory waveform transmitted by bubble CPAP be used to calculate lung compliance (abstract)? 2013 AAP National Conference and Exhibition. https://aap.confex. com/aap/2013/webprogram/Paper22095.html. Accessed October 15, 2014

21. Ho TY, Ou SF, Huang SH, Lee CN, Ger LP, Hsieh KS, et al. Effect of flow rate on delivery of bubble continuous positive airway pressure in an in vitro model. Pediatr Neonatol 2010;51(4):214-218.

22. DiBlasi RM, Zignego JC, Tang DM, Hildebrandt J, Smith CV, Hansen TN, Richardson CP. Noninvasive respiratory support of juvenile rabbits by high-amplitude bubble continuous positive airway pressure. Pediatr Res 2010;67(6):624-629.

23. Van de Kieft M, Dorsey D, Morison D, Bravo L, Venticinque S, Derdak S. High-frequency oscillatory ventilation: lessons learned from mechanical test lung models. Crit Care Med 2005;33(3 Suppl): S142-S147.

24. Yagui AC, Vale LA, Haddad LB, Prado C, Rossi FS, Deutsch AD, Rebello CM. Bubble CPAP versus CPAP with variable flow in newborns with respiratory distress: a randomized controlled trial. J Pediatr 2011;87(6):499-504. 